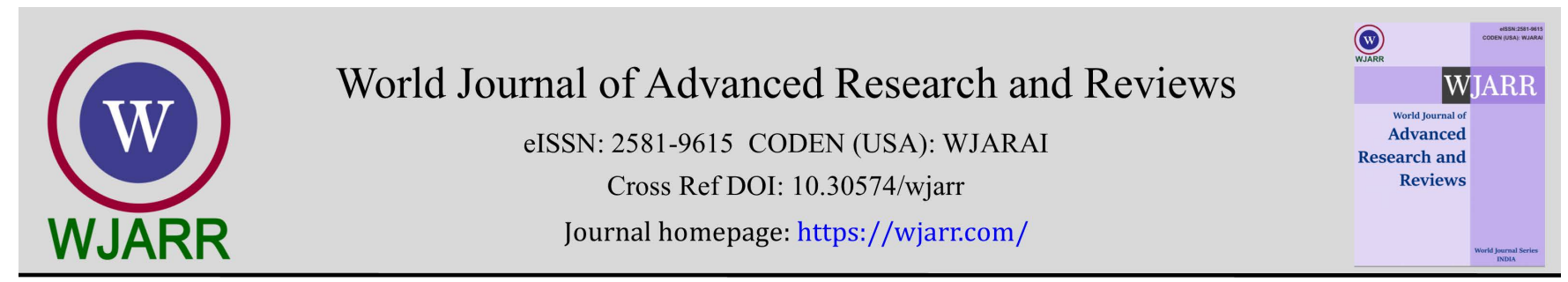

(REVIEW ARTICLE)

\title{
4-dimensional lattice models for the quantum range
}

\author{
Gudrun Kalmbach H.E. *
}

MINT, PF 1533, D-86818 Bad Woerishofen, Germany.

World Journal of Advanced Research and Reviews, 2021, 12(01), 175-181

Publication history: Received on 03 January 2021; revised on 01 February 2021; accepted on 03 February 2021

Article DOI: https://doi.org/10.30574/wjarr.2021.12.1.0003

\begin{abstract}
Some finite subspace models L are presented for quantum structures which replace the use of countable infinite Hilbert


vectors of $\mathrm{H}$ in 24 are drawn as four points on an interval. Blocks can overlap in one or two atoms. Different kinds of operators can map one block onto another and interpretations are given such that subspaces can carry on their base vector tuple real, complex or quaternionic numbers, energies, symmetries and generate coordinate lines. Describing states of physical systems is done using $\mathrm{L}$ and its applications for dynamical modelling. They don't need the infinte dimensional vectors of $\mathrm{H}$. L has in the first model 11 blocks and 24 atoms (figure 1). They correspond to the 24 elements of the tetrahedral S4 symmetry. S4 arises from a spin-line rgb-graviton whirl operator with center at the tip of a tetrahedron and a nucleon triangle base with three quarks as vertices. The triangles factor group D3 of S4 is due to the CPT Klein normal subgroup Z2 x Z2 of S4. It has a strong interaction SI rotor for the nucleons inner dynamics which is used for integrating functions, exchanging energies of nucleon with its environment and setting barycentrical coordinates in the triangle. At their intersection B as barycenter sets a Higgs boson or field the rescaled quark mass of a nucleon. Each factor class of one element from D3 assigns to it a color charge, a coordinate, an energy vector and a symmetry. Symmetries attached can be different according to interactions involved. Every atom of L has then a specific character with different properties. Three characters are added to octonian base vectors, listed by their indices as $\mathrm{n}=$ $0,1, \ldots, 7$, and named for the atoms of $\mathrm{L}$ as na, $\mathrm{nb}$, nc. The structure and element attributes of the finite subspace lattices $\mathrm{L}$ are desribed in many examples and models which technical constructed run macroscopically. Several models are described below.
\end{abstract}

Example, the color charge whirl as rgb-graviton projection operator maps the block 2c3b5a6a to 0a1a2a3a. The symmetries change dimension from $3 \times 3$ - to $2 \times 2$-matrices. From SU(3) are $\lambda 1$ on $3 b$ mapped to the SU(2) $x$-coordinate Pauli matrix $\sigma 1$, from $\lambda 2$ on 5 a to $\sigma 2$ y-coordinate and from $\lambda 3$ on 6 a to $\sigma 3$ z-coordinate of real Euclidean space $\mathrm{R}^{3}$. The $\mathrm{SU}(3)$ matrices have complex $\mathrm{w} 3=\mathrm{z}+\mathrm{ict}$,

$\mathrm{w} 2=(\mathrm{iy}, \mathrm{f}), \mathrm{w} 1=(\mathrm{x}, \mathrm{m})$ coordinates. In figure 3 is shown how a rotation of two proton tetrahedrons for fusion changes the two linearly independent wj vectors to the 1-dimensional $\mathrm{x}, \mathrm{y}, \mathrm{z}$ base vectors. In deuteron then on one coordinate line sit with Cooper paire $\mathrm{u}$-d-quarks at the ends the Heisenberg coupled energy or space vector rays 15 (x,m), m mass measured in $\mathrm{kg}$, $\mathrm{x}$ in meter, 23 (iy,E(rot)), E(rot) rotational energy measured in Joule J, y in meter, 46 (ict,f), t time measured in seconds,

$\mathrm{f}=1 / \Delta \mathrm{t}$ frequency s inverse time interval measured in $\mathrm{Hz}$. The six color charges are red $\mathrm{r}$ on $+\mathrm{x}$ as octonian coordinate 1 , green $\mathrm{g}$ on $+\mathrm{y}$ as 2 , blue $\mathrm{b}$ on $-\mathrm{z}$ as 6 , turquoise on $-\mathrm{x}$ as 5 , magenta on $-\mathrm{y}$ as 3 , yellow on $+\mathrm{z}$ as 4 ..

Keywords: Lattice; Projectivity; Heegard Decomposiions

\footnotetext{
* Corresponding author: Gudrun Kalmbach; E-mail: mint-01@maxi-dsl.de MINT, PF 1533, D-86818 Bad Woerishofen, Germany 


\section{The first L lattice, Fano memo, Physics atoms and Projectivity}

The subspace lattice of $\mathrm{H}$ is orthomodular where the orthogonality comes from splitting $\mathrm{H}=\mathrm{U}+\mathrm{U} \perp$ for closed subspaces $\mathrm{U}$ and its orthogonal space. A vector $w \varepsilon H$ is written as $w=v+v^{\perp}$ with $v \varepsilon U$ and $v^{\perp} \varepsilon U \perp$. The orthogonality $U=U \perp \perp$ generates maximal Boolean subspace lattices $\mathrm{B}$ in $\mathrm{H}$ which intersect settheoretical in Boolean subspaces. The projections $\operatorname{pr}_{B}: \mathrm{H} \rightarrow \mathrm{B}$ are collected in maximal sets of commuting operators and commuting in $\mathrm{L}$ is derived from this. From $\mathrm{H}$ is taken the orthomodular law which holds for the closed subspace lattice of $\mathrm{H}$. Projective geometrical versions are the modular law which corresponds to the fact that in projective spaces any two lines have a point in common, there are no paralelles. The finite model L with 24 base vectors, coming from 8 octonian vectors in figuer 1 shows a possible solution for the 11 blocks. The 4-cycle of blocks requires by the lattice algebra for join, meet and orthocomplementation that it has inside an astroid with 4 blocks. 3-cycles are not existing.

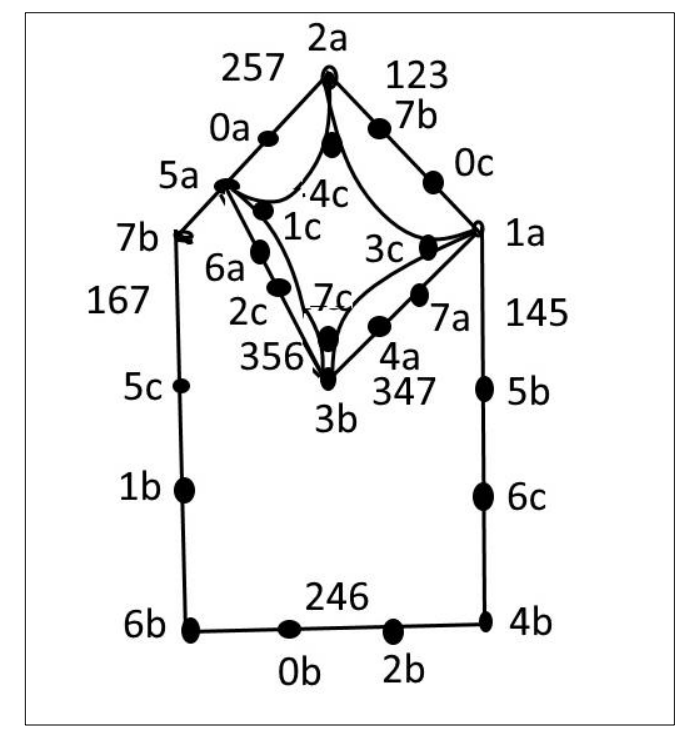

Figure 1 blocks of $\mathrm{L}$ and their atoms, marked on blocks are the 3-dimensional GF Gleason frame bases of the Fano memo for measuring Gleason operators $\mathrm{T}$ which can change metrics in $\langle\mathrm{wT}, \mathrm{w}\rangle$

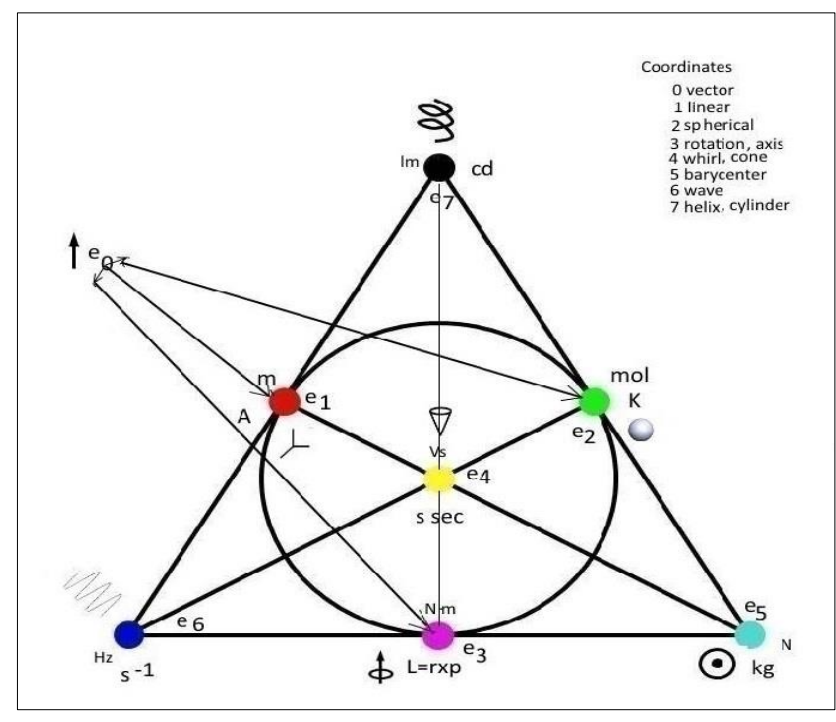

Figure 2 Fano memo without the $\mathrm{e}_{0}$ octonian base vector (at left), the vectorial base triple 123 is for spin and space xyzcoordinates 1, 145 for an electromagnetic EM triple 4, 167 for the electromagnetic interaction EMI 7, 246 for heat 2, 257 for mass , Higgs and barycenters 5, 347 for rotational energy 3, 356 for the SI rotor 6; the measuring GF's allow for their weight no rescaling but rotations of their base on the unit sphere $S^{2}$; extending their base to the 4-dimensional bases of some $\mathrm{L}$ means that the new coordinate allows a restricted projective norming by numbers $b$ with $|\mathrm{b}|=1$; spin for instance can turn up/down by using the parity P operator 
Logos are added for the single listed force vectors: 1 sets orthogonal base triples for the GF, 2 sets volume bubbles for phonons in spacetime with heat entropy inside, 3 sets orbits for roational sytems about a center B in a plane and an orthogonal rotation axis through B, 4 sets conic magnetic field quantums, also for conic rotating spin vectors, 5 sets barycenters for mass systems, 6 sets quantized helix lines winding around a circular cylinder where only full windings are stored as energy, 7 is extended to a Kaluza-Klein U(1) circle for EMI waves, described in form of the complex exponential function $\exp (\mathrm{i} \varphi)$ by adding a projective stereographic point at infinity. Only its real projection cos $\varphi$ is observable in spacetime. Its real derivative $\sin \varphi$ is obseved as waves speed and the second derivative is for the EMI force. The GF are listed at blocks which use them as 3-dimensional bases in figure 1. The astroid $0 \mathrm{a} 1 \mathrm{c} 2 \mathrm{a} 3 \mathrm{c} 4 \mathrm{c} 5 \mathrm{a}$ - $\mathrm{c}$ has no GF's, 6a is a base vector in its outer 4-cycle. This shows that in a partial block structure all octonian coordinates are present. This does not mean that more GF's can be invented, SI with SU(3) matrices has also 7 GF's. 126 as rgb can be a graviton GF which presents the neutral color charge of nucleons.

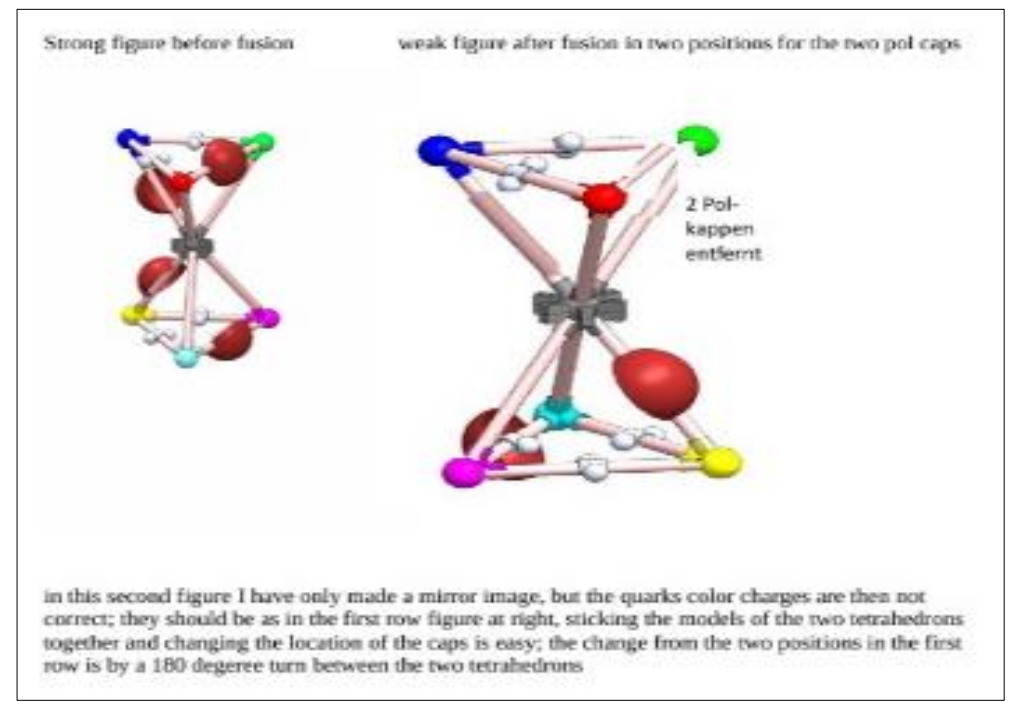

Figure 3 two Cooper paired protons at right with the nucleon triangles parallel; the upper $r g b$-graviton rotates, emitting for fusion a positron and neutrino; then at right a neutron and proton are paired with u-.d-quark pairs at the ends of the three xyz-coordinate axes; the central projection shows the two triangles forming a hexagon

These Cooper pairs form atomic kernels where the common center of the nucleons is split and the two nucleons are in isospin and exciton interactions in contact. If their distance gets too large, nucleon decays occur.

In the astroid the modular law holds. For its four blockx projective $\mathrm{P}^{3}$ spaces can be assumed with coordinates [x,y,z,w]. Many orbital shapes are due to the normal forms of the $\mathrm{P}^{3}$ subspaces $\mathrm{P}^{2}$ as circle, ellipse, parbola, hyperbola, pairs of intersecting lines and 2-dimensional normal quadratic forms are for spheres, cones, 1-sheeted hypebolas which close to the Hopf torus for EM at projective infinity by a circle or pinched torus where an EMI cylinder is closed by a point at infinty. The shapes arise through projective correlations as set of points which lie on its dual hyperplane. Quadratic forms give rise to metrical measures wher operators $\mathrm{P}$ are applied to vectors in $<\mathrm{xP}, \mathrm{x}\rangle$.

Minkowski metric is a spacetime example, replacing Euclidean metric. It uses the light cone as quadric. Projective correlations duality allows to change dimensions of systems and for $\mathrm{P}^{2}$ is important the use of nonorientable Moebius strips where spin-like GF's can rotate on closed central circles and change their up/down normal direction.

\section{Heegard decompositions}

In the first section an example for fusion and the strong nuclear interaction in nucleons is discussed (figure 3). A similar setting is now described for the weak interactions decays of weak bosons. 


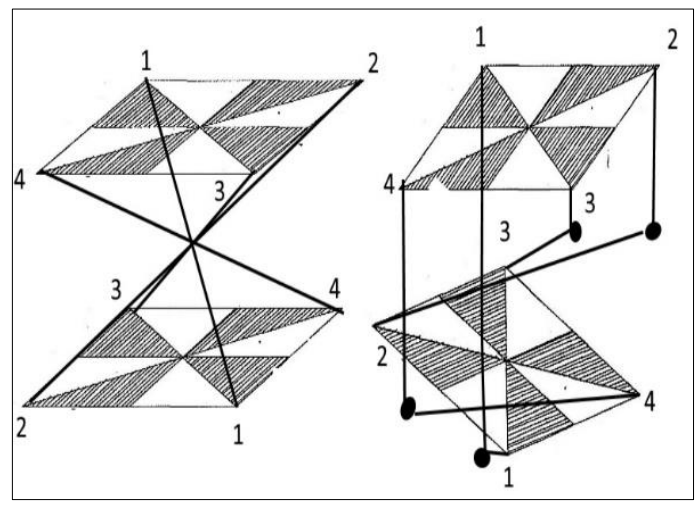

Figure 4 magnetic group as condensor plate; as vertices are suitable the 4-cycle vertices $0 \mathrm{a} 2 \mathrm{a} 3 \mathrm{~b} 5 \mathrm{~b}$, distributed to the attached numbers 1234 for the spacetime coordinates; the spherical coordinates 023 are used and 5 sets a new center for the part at left where Higgs can add a mass scalar for the system at left; at left two particles generated by a weak boson or a hyperphoton decay in form of Cooper pairs electron-antineutrino, positron-neutrino, two photons, for instance are joined at left to the weak boson and splitted at right in form of the Heegard decompositions of the Hopf $S^{3}$ sphere into the two particles which have no common barycenter

Before the second lattice $\mathrm{L}$ is described, the decaying process is presented. A u-quark decay in figure 3 at left is replaced by setting masses differently in a weak boson W. A Higgs boson sets in W three cores for mass charges at rest. One is in the next figure shown as core of the torus. A second core exists for the torus n, not drawn in this figure which belongs to a second similar lepton as the one torus shown in this figure at right. The first core for one lepton e is in the xy-plane, the second in the xz-plane. For $W$ there exists in the yz-plane a similar core, carrying the W mass. If the three Schwarzschild radius circles are shown on a sphere $S^{2}$, they are equators in the three equatorial $x^{-}, x^{-}, y^{-}-$planes. The three masses belong to a kg-GF 056 which carry like a spin GF these weights at their three base vectors. For the W decay, a Higgs boson annihilates the yz-plane core of the $W$ boson and sets two $B_{j}, j=e, n$ kg-GF, one for the two e,n leptons as $\mathrm{W}$ decay products (parts at right). For the electrical charged lepton, $\mathrm{B}_{\mathrm{j}}$ is at rest. In case a special relativistic rescaling is changing the e lepton between three states with the three possible masses attached, this means that the group speed of its matter wave package is also changed. There is no oscillation as in the case of $n$. This is a superpostion of three neutral leptons and in time intervals the observable vector of $\mathrm{B}_{\mathrm{n}}$ is exchanged by a rotation. There is only one wave packege for the three neutrinos, also in this case the group speed of it is changed when the observable mass vector is changed. This is similar to the change of a spin direction for instance observed in the Stern-Gerlach experiment. In the Copenhagen interpretation for quantum measures, only one GF base vector is observable and the other two GF vectors are undetermined. The e,n particles are independent and move in different space directions on their world line. The neutrinos wave package can have a GF with quaternionic $\mathrm{q}=(\mathrm{a}, \mathrm{b}, \mathrm{c}, \mathrm{d})$ coefficients where one neutrinos properties are numerically listed as scalars a for its neutral charge, b for an oriented eigen-rotation clockwise cw or mpo counterclockwise, c for its kg-mass (different for the three neutrinos), d for its momentum. The Heegard decomposition of $\mathrm{W}$ is shwon in figure 6.

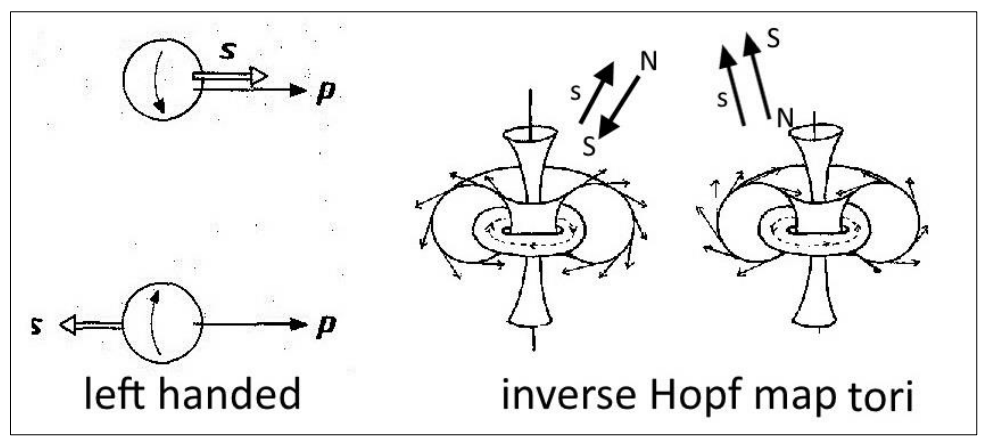

Figure 5 at left the two helcitiy left handed or right handed space rotations of neutral leptons where the momentum changes its direction to the spin vector; at right two Hopf $S^{3}$ equipotential and field lines presentations for the leptons where only one of the two leptons field lines are shown 


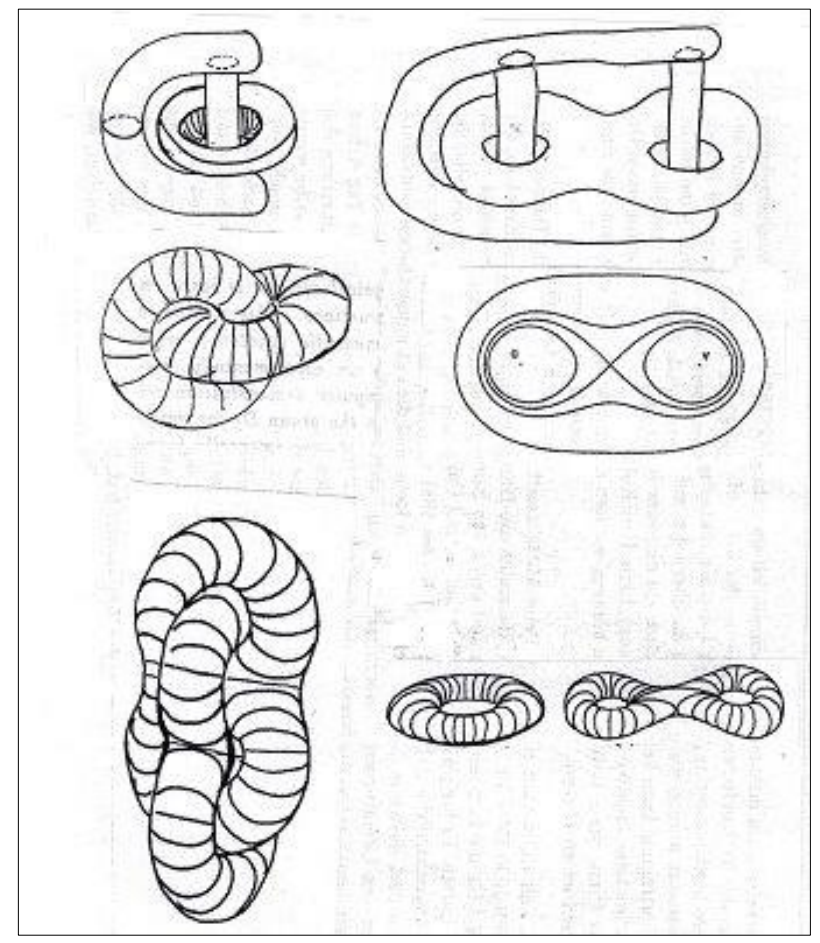

Figure 6 Heegard decompositions of $\mathrm{S}^{3}$, at left the two particles are joined as a W boson; in decay the first line postulates how the two joined particles separate into two particles; the tori of genus 1 ae for two leptons, for instance an electron and an antineutrino wave package, the brezels of genus 2 are for two quarks joined or generated; the Schwarzschild radius core of leptns is for them replaced by a 1-dimensional lemnidcate: the two cases for cores are due to two frequencies in the Lissajous setting; for getting from two orthogonal hitting frequencies, they are in proportion 1:1 for the torus and 1:2 for the brezel

In a 4-dimensional lattice $\mathrm{L}_{\mathrm{w}}$ diagram for $\mathrm{W}$ decompositions there are other $\mathrm{GF}^{\prime} \mathrm{S}$ important which may have a different interpretation as the Fano GF's list for L. It is not necessary to rename the triples: 257 is for $5 \mathrm{~kg}$ GF's in three versions $257_{\mathrm{w}}$ for $\mathrm{W}, 257_{\mathrm{e}}$ for e, $257_{\mathrm{n}}$ for $\mathrm{n} ; 347_{\mathrm{cw}}\left(347_{\mathrm{mpo}}\right.$ ) is for the $3 \mathrm{cw}$ (mpo) orientations, $347_{\mathrm{w}}$ for the rotation in figure 4 from left to right; for 1 it is $123 \mathrm{w}, 123_{\mathrm{e}}, 123_{\mathrm{n}}$ charge; for 4 it is $145 \mathrm{w}$ for a $\mathrm{W}$-momentum, 145e for the magnetic momentum, $145_{n}$ for momentum; for 7 it is $167_{j}$ for wave descriptions, if existing for $\mathrm{j}=\mathrm{e}, \mathrm{n}$ and for $\mathrm{j}=\mathrm{W}$ it can be used for its $S^{3}$ geometry; for 2 it is 246 ; for three entropies, $\mathrm{j}=\mathrm{W}$ inside $S^{3}$ or its Hopf sphere $S^{2}$ bounded space volume, where the second case $\mathrm{j}=\mathrm{e}, \mathrm{n}$ is applied to the two leptons entropy inside their Hopf $\mathrm{S}^{2}$ projection; for 6 it is $356_{\mathrm{j}}$ for three frequencies, angular for e,n eigenrotations as 356e $356 \mathrm{n}$ and $356 \mathrm{w}$ is for Higgs bosons transforming inner speeds, interactions into mass rescalings for the setting of the three $\mathrm{kg}_{-} \mathrm{GF}^{\prime} \mathrm{s}$ in 1 . The triple notation with indices $\mathrm{W}$,e,n replace the octonian base vectors indices a,b,c for L. For Lw the projective correlation duality is applied where the GF triples are replaced by their listed number 1 for 123,2 for 247,3 for 347,4 for 145,5 for 257,6 for 356,7 for 167 . For the octonian coordinate 0 a new $\mathrm{GF}$ in three $\mathrm{j}=\mathrm{W}, \mathrm{e}, \mathrm{n}$ form $037_{\mathrm{j}}$ is postulated where 0 is for the $\mathrm{G}$-compass needle $\mathrm{e}_{0}, 7$ for its disk circumference $U(1)$ and 3 is for a leaning angle of $e_{0}$ towards the $\mathrm{z}$-coordinate of space. For $\mathrm{j}=\mathrm{W}$ it makes the leaning angle in figure 5 right of spin towards the vertical rotation axis; for $\mathrm{j}=\mathrm{e}, \mathrm{n}$ it makes the aligned change of two GF's spin and $145_{\mathrm{j}}$ in superposition where for e spin and magnetic momentum are aligned and for $\mathrm{n}$ spin and momentum. The correlations duality $3 \rightarrow 1$ dimension is possible in 5 dimensions where the four spacetime coordinates is added a projective coordinate in $[\mathrm{x}, \mathrm{y}, \mathrm{z}, \mathrm{ict}, \mathrm{w}]$. With the fomrer replacement, $\mathrm{L}_{\mathrm{w}}$ needs no new L figure 1 to be redrawn, except that the listing of GF's has also be replaced by their associated coordinate numbers. There can be other L figures, which is let as a research project.

\section{Research Projects}

Figure 1 has beside the 4-cycle, a 5-cycle and a 6-cycle. The 4-cycle has 8 atoms. In vectorial notation it has an addition, but multiplications are different according to their use for interactions, spaces, functional integration and differentiation. The author refers to the references and the use of tensor and spinor multiplications in physics. For the vertices of the 4 -cycle, the lines base vectors $0 a, 3 b, 4 a, 5 a$ it can postulated that they arise for energy lines in a projective 
$5 a 6 a$ energy plane as mass-frequency transformations for the line $\mathrm{mc}^{2}=\mathrm{hf}$. Another projective plane can be $0 \mathrm{a} 7 \mathrm{a}$ for a postulated G-compass (see [3]). 3b is for the SI rotor GF 3b5a6a. 5a can be for the mass GF 257. Other interpretations are open for research. 4-dimensional octonian subspces can be $0123,0257,0347,2346$. Adding 0 can mean that in 0123 strong interactions spherical coordinates $0 \mathrm{r}$ (r radius), $2 \varphi, 3 \theta$ are used when the $r g b$-graviton projection changes them to linear weak/EM Euclidean $x=r \cdot \cos \varphi \cdot \sin \theta, y=r \cdot \sin \varphi \cdot \sin \theta, z=r \cdot \cos \theta$ with $r^{2}=x^{2}+y^{2}+z^{2}$ for the metrical distance. In 0257 it can mean that Higgs bosons have a toroidal surface with a 7 circle and a Schwarzschild radius Rs closed circle for 0,5 can set its mass on the core circle of the torus. In 0347 for rotations also 3 is projective closed to a circle for rotational orbits and 4 is a torus axis, 0 can change radial distances.

In 2346, phonons 4 and 6 act for changing inner speeds in a spacial volume bubble having a $23 \mathrm{~S}^{2}$ surface where matter energies generates pressure on $\mathrm{S}^{2}$.

In the 5-cycles the modular law fails, only the weaker orthomodular law holds. As a logical application quantum philosophers are asked to revise their paradoxies since modus ponens, implication are only valid in blocks but fail as logical tools in the 5-cycles. Deductions are not valid for the quantum range. For field presentations in 5-dimensions are useful 1a2a4b6b7b, also in spherical closed form for the unit sphere $S^{5}$ geometrical factor of SU(3) as space for a new fiber bundle, similar to the Hopf fiber bundle. In both cases, the fiber is $\mathrm{S}^{1}$ from 7 . 1a3b4b5a6b7b in 6 dimensions is missing the polar $\varphi$ coordinate. This norms projective, also in the fiber bundles and GF's through its $\exp (i \varphi)$ use the linear spaces to projective spaces. The bundle map g for $\mathrm{S}^{5}$ maps it to a complex 2-dimensional space $\mathrm{CP}^{2}$ with a bounding Riemannian unit sphere $S^{2}$, the Hopf map projects spacetime to space and $S^{3}$ to $S^{2}$ as complex line. $C P^{2}$ are inner spaces for atomic kernels, nucleons, deuteron. They are observed like heat bubbles in spacetime, but have their own inner dynamics. Moebius transformations are added to the symmetry U(1)xSU(2)xSU(3) for icluding gravity actions to the standard model of physics. 145, 246, 167 are listed as GF's on the 6-cycle beside the ones on the 4-cycle. EMI coordinates use EM/WI coordinates with an extended 6-cycle subspace 1a2a3b4b7b. Empty spacetime is extended by $5 \mathrm{a} 6 \mathrm{~b}$ as an Einstein energy plane to a complex 3 -dimensional space $\mathrm{C}^{3} 123456$. $0 \mathrm{a} 7 \mathrm{~b}$ can serve for a projective extension to octonians. The complex cross product adds these new coordinates from spacetime coordinates $\left(\mathrm{z}_{1}, \mathrm{Z}_{2}\right) \varepsilon \mathrm{C}^{2}$ to $\mathrm{z}_{3}=56=\mathrm{z}_{1} \times \mathrm{z}_{2}, \mathrm{z}_{4}=07=\mathrm{z}_{1} \times \mathrm{z}_{2} \times \mathrm{z}_{3}$. Open for research are further uses of the 4-,6-cycles.

The doubling of quaternions to octonians is shown in the model of figure 4, similar to the deuteron model (figure 3). Two quadratic magnetic group condensor plates ar set parallel in figure 4 and the diagonals between them are the four spactime coordinates. A 45 or 135 degree rotation of the upper plate split the diagonals into two complex vectorial rays such that the four lines are replaced by four complex planes for octonian coordinates. EMI 167 is added to the other three basic forces GR, SI, WI (gravity, weak, strong interactions) when atoms can send out light. Accoustic heat whirls 246 occur and the GF 145 is for the full use of EM. At the center of the part at left sits a weak boson and has at the quadrats vertices added replacements of quarks in a nucleon triangle. Repeated is: 1 can carry in the upper quadrangle electrical charge e, 4 magnetic momentum, 2 sets a rotational mpo + or cw - clockwise orientation on an induction 3 ircle containg e, and similar settings are for the neutral leptons on the lower quadrangle where momentum is substituted for the magnetic momentum and their helicity.

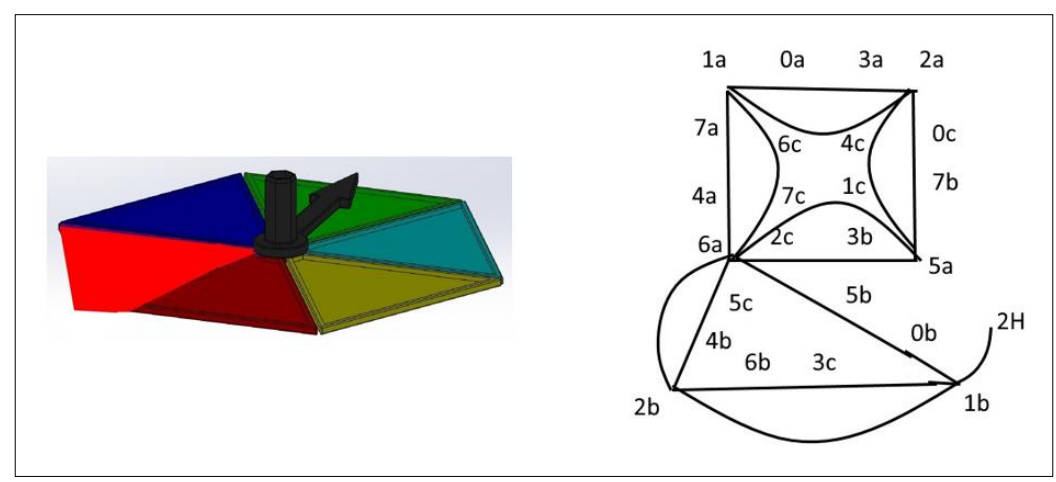

Figure 7 G-compass, also for other nth roots of unity available, here $n=6$ for discrete turns of the needle (left); for the Lg lattice at right the $r g b$-graviton having a quark triangle and a needed central block $1 \mathrm{~b} 2 \mathrm{~b} 4 \mathrm{H} 6 \mathrm{a}$, a coordinate $4 \mathrm{H}$ is added for Higgs or a chronon

For further research is suggested to look at the list of GF's. It can be extended for quaiparticle GF's where in another article of the author was made some comments. Here is added: $126 \mathrm{WI}$ or $123 \mathrm{SI} \mathrm{GF}$ for rgb-graviton whirls, $056 \mathrm{~kg}$-GF, 037 for the solitons or G-compass, the list from the Fano memo where 123 is for spin, 246SI can be for a chronon as 
time-quasiparticle, 067 for anyons, 257SI for Higgs bosons, 246 for phonons, 356 for helicons, 347 for orbitons or rotons, 167 for photons, 027 for polarons, 047 for solitons, 017 for wrinkletons, 047 for trions, and so on. There is then a need to construct meaningful models like the nucleon tetrahedron in figure 4 for 126 and also a need for extending the triples to 4-dimensional lattices $\mathrm{Lj}_{\mathrm{j}}$ as in figure 1 and 7.

\section{Conclusion}

As research project, 4-dimensional real or complex Hilbert spaces are suggested for the quantum range.Their Heegard decompositions are discussed, geometrically the model is projective and it uses the measuring apparati of Gleason operators and their frames. They obey the Copenhagen interpretation.

\section{References}

[1] Kalmbach, G. (1983): Orthomodular Lattices. - London New York: Academic Press 390p.

[2] Kalmbach H E, G 2019: MINT-Wigris. - MINT Verlag Bad Woerishofen.

[3] Kalmbach H E, G 2020: Gravity with color charges. - Preprint to appear.

[4] Kalmbach H E, G 2020: MINT (Mathematik, Informatik, Naturwissenschaften, Technik), Volume 1-65. - Bad Wörishofen: MINT Verlag. 